\title{
Characterization of a new class of blue-fluorescent lipid droplet markers for live-cell imaging in plants.
}

\author{
Soujanya Kuntam ${ }^{1}$, László G. Puskás², Ferhan Ayaydinn ${ }^{1, *}$ \\ ${ }^{1}$ Cellular Imaging Laboratory, Biological Research Center, Hungarian Academy of Sciences, Temesvári \\ krt. 62. H-6726, Szeged, Hungary. \\ ${ }^{2}$ Laboratory of Functional Genomics, Institute of Genetics, Biological Research Center, Hungarian \\ Academy of Sciences, Temesvári krt. 62, Szeged 6726, Hungary
}

*Corresponding author; email: ferhan@brc.hu, Tel: +36 62599665 Fax: +36 62432576

\begin{abstract}
:
Key message: The present work demonstrates the use and advantages of novel, live cell permeable, Lipid droplet localizing, non toxic, blue fluorochromes for use in live plant cells.

Abstract: Lipid droplets (LDs) are ubiquitous components of both animal and plant cells. They consist of a core of neutral lipids surrounded by a monolayer of phospholipids, glycolipids and/or sterols with embedded amphipathic proteins. Although initially considered to be simple energy depots, they have recently emerged as organelles that serve important regulatory functions. Here we report three new fluorochromes as markers for LDs in plants. These bright blue fluorochromes with their unique spectral properties can easily be combined with other green and red fluorescent reporters for multicolor fluorescence imaging. The fluorochromes are non-toxic and photo-stable. All in all, they represent a reliable tool to use, for the investigation of dynamic LD biology within living plant cells using fluorescence microscopy.
\end{abstract}

Key words: oil body, lipid droplet, fluorescent dye discovery, in vivo staining, confocal laser scanning microscopy.

$\begin{array}{ll}\text { Abbreviations: } \\ \text { DMSO } & \text { Dimethyl sulfoxide } \\ \text { ER } & \text { Endoplasmic reticulum } \\ \text { LD } & \text { Lipid droplet } \\ \text { PI } & \text { Propidium iodide } \\ \text { TAG } & \text { Triacylglycerols }\end{array}$

\section{Introduction:}

Plant cells store neutral lipids such as triacylglycerols (TAG) in distinct cytosolic organelles called Lipid droplets (LDs), also referred to as lipid bodies/droplets, spherosomes or oleosomes (Murphy, 2001). LDs typically are made up of a neutral lipid core surrounded by a monolayer of phospholipids, glycolipids and/or sterols, with a series of proteins bound to/embedded in their surface (Chapman \& Ohlrogge, 2012; Purkrtova et al. 2008). LDs in plants, as in all other eukaryotes, are thought to arise from the endoplasmic reticulum (ER), where they are assembled in specialized ER microdomains. LD formation begins with the accumulation of neutral lipids such as TAG between the two leaflets of the ER 
bilayer, followed by the expansion and eventual release of small LDs into the cytoplasm, which mature into larger droplets under the control of specific proteins (Gidda et al., 2011; Chapman et al., 2012). During most of the $20^{\text {th }}$ century, LDs were regarded as specialized storage organelles limited to specific cell types such as cotyledon, mesocarp or scutellar cells (Herman, 2008; He \& Wu, 2009; Baud \& Lepiniec, 2010). But in the past years, a lot of evidence has emerged pointing towards the presence of dynamic LDs in different cell types such as, leaf mesophyll cells (Lersten et al., 2006; Slocombe et al., 2009), roots (Murphy, 2001) and meristems (van der Schoot \& Rinne, 2011). Also, recent studies indicate, that the role of LDs in plants extends well beyond their role as simply static depots for carbon storage in seeds. Their role includes stress response and pathogen resistance (Coca \& San Segundo, 2010), dormancy-release in shoot epical meristems (Rinne et al., 2001), dormancy process in root nodules, photoperiod signaling (Grefen et al., 2008), lipid homeostasis, hormone metabolism/signaling and a specialized role in anther development (Hsieh \& Huang, 2004; Hsieh \& Huang, 2007). But a lot still remains to be uncovered in the area of LD protein and lipid composition as well as LD transport, mechanism of protein targeting, assembly and regulation. Live cell analysis is thus required to unravel the dynamic regulation of this important organelle.

In recent times, progress in genomics, proteomics and technical advances in cell biology, have helped in broadening our understanding of the nature and function of LDs. Techniques such as genome/transcriptome sequencing of whole organism/specific cell types, generating gene knockout/overexpression lines and developments in mass spectrometry have enabled researchers to extend their knowledge of LD composition, function and remodeling in live cells (de Kroon, 2007). But some of the most important insights have come from the use of new imaging techniques. They have helped us understand the real time dynamics of LDs in live cells and following such behavior during processes such as inflammatory responses (Melo et al., 2011). Examples of such imaging techniques include: vibrational imaging of LDs in live fibroblast cells (Nan et al., 2003), quantitative electron microscopy (Cheng et al., 2009) and confocal reflection microscopy (Gáspár \& Szabad, 2009).

However, in the past few years, there have been particular advances in the use of live imaging systems including live microscopy (Digel et al., 2010, Somwar et al., 2011), time-lapse adaptive harmonic generation microscopy (Watanabe et al., 2010) and vital staining in combination with fluorescence activated cell sorting (Cooper et al., 2010). Such imaging tools in combination with photostable genetically-encoded fluorescent proteins can be used in multiplexed tracking of protein remodeling on LDs (Tsien, 2009). Photo-switchable fluorescent proteins as well as fluorescent timers can be used for the quantitative assessment of LD protein dynamics (Terskikh et al., 2000; Chudakov et al., 2007). Protein conformational changes or protein-protein interactions relevant to LDs can be observed using fluorescent sensors, such as those based on fluorescence resonance energy transfer (FRET) (Giepmans et al., 2006). Combining a reliable LD marker with these tools can create a versatile scheme for investigating LD biology. On the other hand, most of the commercially available live cell, LD dyes are limiting in their ability to penetrate plant cell wall and those that are permeable, such as Nile Red and BODIPY 493/503 have certain drawbacks. Not only does Nile Red have a broad emission range occupying the orange and red regions of the spectrum, but also its absorption significantly overlaps with widely used green and red reporters such as EGFP and mCherry, hindering the use of most ready-made fluorescent reporters in colocalization experiments (Miquel et al., 2014). Although BODIPY 493/503 (which fluoresces in green) can be combined with red-fluorescent constructs for two-color imaging, its lesser photostability and photoconvertible properties limit its ability to be utilized in long term imaging experiments (Ohsaki et al., 
2010). Even though there are other live cell stainingLD dyes with better photostability, such as BODIPY 505/515 and LD540 (Spandl et al., 2009), they still fluoresce in the green to orange region of the visible spectrum. Recently, a new blue-red emission live cell dye monodanyslpentane (MDH) has also been reported (Yang et al., 2012). Due to its dansyl moiety, this dye shows strong solvatochromatic behaviour and shows blue emission on LDs and red emission $(550-650 \mathrm{~nm}$ ) in aqueous cellular compartments (Yang et al., 2012). This dual behaviour necessitates proper configuration of microscope settings in multicolor experiments. Therefore, the identification of a vital dye that can stain LDs while being spectrally well separated from most green, orange and red fluorescence reporters will be a great asset for live cell imaging in studying LD biology.

Herein, we report three new fluorochromes as markers for LD in live plant cells. All are $405 \mathrm{~nm}$ violet laser excitable, compatible with most conventional fluorescence and confocal fluorescence microscopes and emit in the blue range. Easily combinable with different fluorescent reporters for multicolor live cell imaging, these fluorochromes are highly photostable withstanding multiple successive imaging without photoconversion or fading. We found that the fluorochromes are non toxic and are stable enough for long term analyses. Taken together; our findings demonstrate that these new chemicals provide a novel approach for microscopy analysis of LDs in live plant cells.

\section{Materials and methods:}

\section{Chemicals}

The three chemicals used in this study, Ac-201, Ac-202 and Ac-1041, were in-house synthesized thalidomide analogues (The code "Ac" represents a group of in-house synthesized compounds). 2,6diisopropylphenyl- 4/5-amino-substituted- 4/5,6,7-trifluorophthalimides (AC-201: 4-ethlyamino-; AC202: 5-ethyl-; AC-1041: 4-morpholine-) were synthesized as described previously (Puskás et al., 2010). Unless specified all chemicals were purchased from Sigma (Sigma, St. Louis,. MO)

\section{Plant material}

Rice, Arabidopsis, alfalfa, maize and tobacco suspension cultures were grown in their own growth medium (Kotogány et al., 2010). Suspension cultures were maintained in a shaker incubator at $22^{\circ} \mathrm{C}, 140 \mathrm{rpm}$ and subcultured every week. Arabidopsis seedlings ( $7 \mathrm{~d}$ old) were grown on half strength MS medium with $0.7 \%$ plant agar (Duchefa Biochemie B.V., Haarlem, The Netherlands).

\section{Treatment with fluorochromes}

Actively dividing suspension cells (2-4 days old) were used in all of the experiments except Fig $1 \mathrm{x}$ where $9 \mathrm{~d}$ old culture was used to show cell death in an aging culture. The cell cultures were treated with the chemicals at 1,10 and $50 \mu \mathrm{M}$ for various durations in 4 chambered $35 \mathrm{~mm}$ glass bottom dishes (Greiner Bio-One, Kremsmünster, Austria). Dye labeling of Arabidopsis seedlings were done in microfuge tubes using $10 \mu \mathrm{M}$ Ac chemical for $1 \mathrm{~h}$. DMSO $(1 \%, \mathrm{v} / \mathrm{v})$ was used as carrier control in all experiments.

\section{Co-labeling}

Stock solution of Nile Red is prepared as $100 \mathrm{ng} / \mu \mathrm{L}$ in DMSO and $500 \mathrm{ng} / \mathrm{mL}$ is used to label LDs in colocalization experiments. For viability assays, propidium iodide $(0.45 \mu \mathrm{M})$ was added 5 mins before microscopy imaging.

\section{Confocal laser scanning microscopy}


Imaging was performed using Olympus Fluoview FV1000 (Olympus Life Science Europa $\mathrm{GmbH}$, Hamburg, Germany). Microscope configuration was as follows: Objective lens: UPLSAPO $20 \times$ (dry, N.A: 0.75); sampling speed: $4 \mu \mathrm{s} /$ pixel; line averaging: $2 \times$; scanning mode: sequential unidirectional; excitation: $405 \mathrm{~nm}$ (blue LD dyes), $488 \mathrm{~nm}$ (Nile Red, spectral scan), $543 \mathrm{~nm}$ (PI, Nile Red); maximum laser transmissivity values: $2 \%(405 \mathrm{~nm}), 5 \%(488 \mathrm{~nm})$ and $50 \%(543 \mathrm{~nm})$; emission filters: Blue: 425-475 nm; Red: 555-655 nm; Chlorophyll: 650-750 nm. Images were pseudocolored using Olympus Fluoview software (version $4.0 \mathrm{c}$ ). Spectral imaging was performed by using lambda scan mode of Olympus software (20 nm intervals, $10 \mathrm{~nm}$ apart). Leica SP5 AOBS (Leica, Heidelberg, Germany) confocal laser scanning microscope was used for images in Fig. 5. Single optical sections or successive 3D optical sections of seedlings were taken using HCX PLAPO 63x (N.A 1.2) water immersion objective and analyzed with Leica Application Suite Advanced Fluorescence (LAS AF 2.5) software. "Transparent" 3D projection mode of LAS AF software was used to reconstruct the 3D image using 396 optical sections of a $145 \times 145 \mu \mathrm{m}$ square region encompassing a $39 \mu \mathrm{m}$ thick cotyledon region. Another set of 396 sections were captured from a smaller cotyledon area (x, y, z: 145 x 24 x $39 \mu \mathrm{m})$ and scanned using faster scanning parameters to be used in $\mathrm{Y}$-axis projection images. Ac chemicals and chlorophyll fluorescence were detected between 418-536 nm and 650-750 nm, respectively.

\section{Results:}

\section{Localization and toxicity of Ac chemicals on plant suspension cultures}

In our earlier study, we have discovered in-house synthesized thalidomide analogs localizing to lipid droplets on human cancer cells (Puskás et al., 2010). Being blue fluorescent and plasma membrane permeable, these dyes are attractive candidates as fluorescent reporters for Lipid droplet (LD) studies in plant cells. However, not all animal cell permeable dyes are suitable for use in plant studies due to presence of plant cell walls as physical barriers. Lower acidity of plant cell walls and plant culture mediums can also negatively affect fluorescence emission properties of such chemicals in plant experiments. Moreover, biocompatibility and toxicity of these chemicals may also be different for plant cells. In addition to Ac-202 and Ac-1041 chemicals (Puskás et al., 2010), we have also included Ac-201 (a positional isomer of Ac-202) to test suitability of these chemicals as LD probes in plant cells. Using confocal laser scanning microscopy and fine rice suspension cultures (Oryza sativa L. japonica cv. 'Unggi 9'), we have tested cell wall penetration and labeling efficiency of these chemicals. Various concentrations and treatment durations were screened using live cell cultures.

All three chemicals penetrated suspension cultures of rice and displayed bright spotty signals which were uniformly distributed all over the cytoplasm (Fig. 1). Intracellular blue spotty signals were detectable even at $1 \mu \mathrm{M}$ concentration for Ac-201 and Ac-202 following incubation periods as low as 15 min (Fig. 1 a, c). Ac-1041 labeling was extremely weak when used at this concentration. One micromole concentration was not sufficient for long term experiments, as the signal intensity diminished after $24 \mathrm{~h}$ of incubation for all three chemicals (Fig. $1 \mathrm{~b}, \mathrm{~d}$, f). Treatment with higher concentrations, such as $10 \mu \mathrm{M}$ and $50 \mu \mathrm{M}$ resulted in faster accumulation of bright signals within 15 mins (Fig 1g, m, i, o, k, q). Ac-201 and Ac-202 were still visible even after $24 \mathrm{~h}$ when used at $10 \mu \mathrm{M}$ concentration (Fig. $1 \mathrm{~h}, \mathrm{j}$ ). Using identical detection settings, Ac-202 showed the brightest signal in the rice culture used in our experiments and at least $10 \mu \mathrm{M}$ concentration was needed for proper detection of Ac-1041 chemical in short term experiments (Fig. 1k). Control cultures treated with the solvent DMSO (1\%, v/v) did not display 
detectable blue autofluorescence in short term or long term experiments (Fig. $1 \mathrm{~s}, \mathrm{t}$ ). The chemicals were added directly into cultures growing in rice culture medium (G1) with a starting pH of 5.6. Age of cell culture or $\mathrm{pH}$ changes of aging medium did not significantly affect labeling intensity or penetration efficiency as we could detect similar labeling efficiency on both young and old cultures. Furthermore, direct addition of chemicals into liquid growth medium did not induce detectable precipitation or background fluorescence even at $50 \mu \mathrm{M}$ concentration (Fig. 1a-r).

To assess the effect of these chemicals on cell viability and plasma membrane integrity, we have incubated chemical-treated cells with propidium iodide (PI), a membrane impermeable, red fluorescent nucleic acid dye. PI immediately enters dead cells and stains nucleic acids bright red due to compromised plasma membranes (Truernit \& Haseloff, 2008). When used at 1-50 $\mu \mathrm{M}$ concentration range, Ac chemicals did not cause significant increase in PI labeling even after $24 \mathrm{~h}$ treatment (see for example Fig. $1 \mathrm{n}, \mathrm{p}, \mathrm{r}$ ). Bright field (transmission) microscopy images of the treated cells were also comparable to control, DMSO-treated cultures, as abnormalities such as cell swelling, shrinking, color change or clumping were very rare in treated cultures (Fig 1, a-t, bright field images). DMSO is used as solvent of Ac chemicals in our experiments and the level of DMSO concentration was always kept at or below $1 \%$ (v/v). In control experiments, at $1 \%$ DMSO concentration, cell viability was not affected significantly (Fig. $1 \mathrm{~s}$, t, red PI labeling). However, 10\% (v/v) DMSO caused significant increase in cell mortality as judged by PI staining of cultured cells (Fig. $1 \mathrm{u}$, v, note numerous red nuclei). Mortality increase due to high DMSO treatment was immediately evident even after 15 minutes (Fig 1u). Similar to 1\% DMSO treated cultures, untreated young cultures showed low level of cell death (Fig 1w). As the culture gets older, the number of dead clusters increase (Fig $1 \mathrm{x}$ ). Therefore, only young cultures ( $2-4 \mathrm{~d}$ old) were used in experiments. Overall, these data suggest, that when used below $50 \mu \mathrm{M}$ concentration and without exceeding $1 \%$ DMSO as final solvent concentration, Ac chemicals do not have significant toxicity issues in plants even during long term experiments.

\section{Ac chemicals and Nile Red colocalization}

In order to evaluate whether the intracellular bright granules highlighted by Ac chemicals are Lipid droplets or not, we have used Nile Red, a red fluorescent, live cell probe for LDs (Greenspan et al., 1985). All three Ac chemicals colocalized completely with Nile Red, suggesting that Ac chemicals are specifically recognizing LDs in plant cells (Fig. 2a-c). In single dye labeling experiments, both blue and red emission range images were captured to assess the degree of channel crosstalk and autofluorescence (Fig. 2d). Ac dyes did not display any signal in red detection range (Fig. 2d, second column, southwest, northwest and northeast sectors) and Nile Red was not detectable in blue detection range that is used to detect Ac chemicals (Fig. 2d, first column, south sector). DMSO (1\%) did not induce any autofluorescence either in blue or red detection ranges (Fig. 2d, first and second columns, southeast sectors).

\section{Spectral emission characteristics of Ac chemicals}

Although dyes such as Nile Red and the BODIPY derivative LD540 (Spandl et al., 2009) are available as live cell LD probes, Ac chemicals present a significant advantage overthese dyes in multi color experiments and in experiments involving green tissues with interfering red chlorophyll fluorescence. To analyze comparative in vivo fluorescence emission characteristics of Ac chemicals, we have used a microscopy technique called spectral imaging also known as lambda scan (Haraguchi et al., 
2002). This imaging technique allows quantitative determination of emission characteristics of a fluorochrome inside a living cell under physiological conditions. Blue-cyan range fluorescence emission spectra of Ac chemicals, yellow-green colored LD540 and orange-red colored Nile Red fluorescence, collected using spectral imaging of stained rice cell cultures are shown in Fig. 3. All Ac chemicals displayed peak intensity at images captured at 460-480 nm detection interval (Fig. 3, spectral image series). Ac-202 displayed a wider emission curve extending into shorter blue wavelengths of the spectrum. Nile Red, on the other hand, displayed emission positioned at the middle of the visible spectrum peaking at 580-600 $\mathrm{nm}$ interval. This broad emission range significantly overlaps with yellow, orange and red fluorescent proteins widely used in in vivo studies (Fig. 3, rectangular labels on chart). Moreover, the emission range of Nile Red also overlaps with chlorophyll autofluorescence of green tissues requiring careful selection of bandpass emission filters (or tuning of spectral detectors) for proper discrimination of LDs and chloroplasts (Fig. 3). Interference with chlorophyll fluorescence should not present a significant issue with LD540 dye which has a narrow emission around $540 \mathrm{~nm}$. On the other hand, emission of LD540 significantly colocalizes with YFP fluorescence and partially overlaps with green (e.g: EGFP) and orange colored probes (Fig 3). Specific detection and discrimination of LD540 dye in multicolor experiments, therefore requires specific filters and/or carefully adjusted microscopy parameters (Spandl et al., 2009). Ac chemicals with their spectrally well isolated blue fluorescence can be confidently and easily isolated form green and red colored dyes and fluorescent proteins without significant crosstalk issues (Supplementary Fig. 1)

\section{Lipid droplet detection on various plant cultures}

Due to differences in cell wall structure, cellular architecture and metabolism, different plant species may display different dye penetration and labeling characteristics. Therefore we tested Ac chemicals on several monocot and dicot plant cultures to assess their behavior. Under identical treatment $(10 \mu \mathrm{M}, 15 \mathrm{~min})$ and image capturing conditions, alfalfa cultures (M. sativa ssp. varia A2) showed the most intense labeling with Ac dyes. (Fig. 4a-c). Ac-201 and Ac-202, but not Ac-1041 displayed high cytoplasmic background in alfalfa cultures. Ac-1041 labeling on alfalfa was composed of fine, discrete spots with minimal background making this chemical more suitable for LD studies in this particular plant culture (Fig. 4c). For all other cultures tested, Ac-1041 (10 $\mu \mathrm{M}, 15$ ') showed detectable but very weak signal (Fig 4f, i, 1 and insets in 1). Maize culture used in our experiments (Z. mays, cv. H1233) displayed a surprisingly high LD heterogeneity when labeled with Ac 201/202 dyes. Several LD rich cells with multiple big and bright fluorescent spots were clearly distinguishable from neighboring cells with minimal LD labeling (Fig. 4 d, e). This striking difference was not due to lack of dye penetration to some cells, as we could see fine Lipid droplets and a faint cytoplasmic dye background in all of the cells (Fig $4 \mathrm{~d}$, e compare with $4 \mathrm{f}$ ). To further provide evidence that the observed heterogeneity in Fig 4 was not due to issues related to single optical sections, we have captured multiple optical z-sections of cell clusters and quantified average fluorescence intensities of individual cells labeled with Ac201 dye (Supplementary Fig. 2 as animated gif image) Fluorescence intensity differences of more than 3 times could be detectable between certain cells (Supplementary Fig 2c). Large cells of tobacco SR1 culture (N. tabacum cv. Petit Havana SR1) displayed very fine LDs equally efficiently labeled with Ac-201 and Ac-202 dyes. Perinuclear region, cytoplasm and cytoplasmic strands were rich in fine LDs. Similar to alfalfa and tobacco cultures, Arabidopsis culture (A. thaliana ecotype Landsberg erecta, MM1) also displayed homogenous LD labeling within a given cell cluster with Ac-201/202 chemicals (Fig. 4 j-1). Presence of bright red chlorophyll signal in this Arabidopsis culture did not present 
emission crosstalk issues as we could clearly isolate spectrally well-separated blue Ac chemical-labeled LDs from red chloroplasts (Fig. 4 j-1, insets).

\section{Lipid droplet labeling on intact Arabidopsis seedlings}

Plant suspension cultures are often made up of relatively small clusters with only a few layers of mostly homogenous cells. On the other hand, tissues and organs of plants with multiple layers of diverse cells present penetration barriers for dyes targeted for use in vivo. To realize the full potential of Ac chemicals as LD stains for plants, we have also tested these chemicals in germinating Arabidopsis seedlings. As shown in Fig. 5, both Ac-201 and Ac-202 dyes were equally suitable for use in various organs and tissues of intact seedlings. Dense root meristem region with multiple layers of actively dividing cells (Fig. 5a and inset), fine root hairs (Fig. 5b), hypocotyl (Fig. 5c) and epidermis of cotyledon (Fig. 5d) were all showing fine spots of Lipid droplet labeling with Ac-201 indicating successful probe penetration and LD labeling. Ac-1041 chemical was equally effective in root hair and hypocotyl region labeling (Supplementary Fig. 3) but in dense and multilayered tissues (such as root tip), it was weaker in intensity as compared to Ac-201/202. As in Arabidopsis MM1 culture, use of blue fluorescent hypocotyl (Fig 5c) and cotyledon regions (Fig 5d, e-g) was particularly advantageous. Efficient penetration and bright fluorescence emission of these dyes also allowed us to use low excitation energies. This is particularly beneficial in 3D confocal optical sections and reconstructions where multiple successive image capturing is needed. Images presented in Fig. 5e and $5 \mathrm{f}$ were prepared by laser scanning of 396 consecutive optical sections without significant dye bleaching (see also Fig $5 \mathrm{~g}$ for additional 396 scans of a subset area). In order to reduce image smearing due to mobile Lipid droplets, we have rescanned a smaller area using faster scanning parameters. Fig $5 \mathrm{~g}$ shows $\mathrm{y}$-axis projection (side view) of the selected area (yellow rectangle in Fig 5e) using additional 396 optical sections from the same cotyledon area. Note that a total of nearly 800 times repeated laser scanning of the same area did not cause significant signal bleaching of Ac-202 fluorescence. As evidenced by these 3D confocal optical sections (Fig. 5f, g), epidermis of Arabidopsis cotyledons contain significantly more LDs than parenchyma region. This difference was not due to lack of dye penetration to parenchyma region as evidenced by the presence of LD labeling between and around the chloroplasts of parenchyma cells in serial optical z-sections (Supplementary Fig. 4, animated gif image showing confocal sections).

\section{Discussion:}

Plasma membrane permeable live dyes discovered and developed in, for example, animal model cultures may not always be practical in intact plant studies due to the presence of the cell wall. For example, blue fluorescent live cell nuclear dyes used in animal cells such as DAPI, Hoechst 33258 and Hoechst 33342 or several fluorescent $\mathrm{pH}$ indicator dyes used in living animal cells cannot be efficiently used in intact live plant cells. Wounding stress-inducing microinjection or protoplastation is required for such cell wall impermeable dyes to be delivered into plant cells in in vivo studies (Fasano et al., 2001). Further, the small number of LD dyes available for use in live plant cells, like BODIPY and Nile Red has certain drawbacks during multicolor imaging. While Nile Red has a very broad emission range interfering with the chlorophyll fluorescence and hindering multicolor imaging (Miquel et al., 2014), BODIPY 493/503 with its lesser photostability and photoconvertible properties limit its use for long term, repetitive imaging (Ohsaki et al., 2010). Despite the presence of other LD dyes with better photostability such as BODIPY 505/515,LD540 (Spandl et al 2009) or blue-red colored dual behaving monodanylpentane 
(Yang et al., 2012) the problem still remains that they have emission in the green, yellow or red region of the visible spectrum. Combined, all these observations call for the identification of a vital LD dye for plants which is spectrally well separated from the majority of the live fluorescent markers and chlorophyll autofluorescence.

In the present study, we have demonstrated that three recently developed lipid droplet localizing fluorochromes serve as robust markers for LDs in living plant cells. We have tested different concentrations of these fluorochromes and found that $10 \mu \mathrm{M}$ concentration gave the best results for both suspension cultured cells and whole seedlings. Long term labeling (24 hours) of cells with these fluorochromes did not cause any significant increase in cell death, even at concentrations as high $50 \mu \mathrm{M}$ (Fig 1). Cell viability was tested using the red fluorescent dye propidium iodide (PI). Apart from being a viability indicator, this dye can also be used as a marker of cell borders in leaf and root tissues (Truernit \& Haseloff, 2008). All three blue Ac chemicals could be used efficiently in combination with PI, once again confirming their advantages in multicolor imaging. LD labeling of the three chemicals was also validated by colocalizing with Nile Red, a well known lipophilic LD marker (Greenspan et al., 1985). Similar to Nile Red, Ac chemicals being lipophilic thalidomide analogues, specifically accumulated in LDs. Further, in vivo fluorescence emission characteristics of the fluorochromes were determined through spectral imaging/lambda scanning microscopy technique (Haraguchi et al., 2002). All three dyes were found to be efficiently excited using $405 \mathrm{~nm}$ laser, commonly available on laser scanning confocal fluorescence microscope setups. All three chemicals displayed peak emission intensity at 460-480 nm detection interval. Although Ac-201 and Ac-202 are positional isomers with very similar chemical structures, their emission characteristics were not identical (Fig. 3). Ac-202 displayed a more asymmetric emission curve with a pronounced shoulder around $430 \mathrm{~nm}$. Ac-201 and Ac-202 also differed in LD labeling intensities when used on rice cultures (Fig 1). Such differences in intensity between Ac-201 and 202 was not detectable on other cell cultures tested. On the other hand, these chemicals displayed high background in alfalfa (ssp. varia A2) cultures, for which use of Ac-1041 displayed better results with discrete LD labeling (Fig 4a). Using less concentrated Ac chemicals on alfalfa resulted in unsatisfactory results (Supplementary Fig. 5). Staining intensity and/or penetration capabilities of Ac-1041 was less favorable in other cell cultures. Nevertheless, spectral emission and labeling intensity differences between these highly similar thalidomide derivatives suggest that additional alternative modifications on the base thalidomide structure may yield even better LD probes with superior characteristics. In addition to celltype specific labeling intensity differences, we have also observed differences in LD content within a given cell cluster. LD labeling in maize (cv. H1233) suspension culture, for example, was surprisingly heterogeneous with certain cells showing elevated LD accumulation (Fig 4d). In these distinctly LD-rich cells of a given cell cluster, either LD synthesis and fusion was upregulated or their breakdown was slowed down due to some yet unknown reason. Highly fluorescent Ac chemicals can also be detected with equipment other than microscopes such as flow cytometer or fluorescence plate readers. It can be very interesting to isolate and characterize these LD-rich maize cells by using flow sorting of stained protoplasts, for example.

Advantages of Ac chemicals were particularly evident in three dimensional microscopy analyses of green tissues. We have shown in this study that in Arabidopsis, Ac-201 and 202 chemicals can penetrate deep multilayered tissues of intact seedlings (Fig. 5). Both underground and aboveground tissues of seedlings showed several LDs stained with Ac chemicals (Fig 5a-d). Repetitive laser scanning during collection of multiple z-axis optical sections on stained cotyledons did not cause significant signal 
fading of Ac chemicals. Using 3D reconstructions of Ac-202 stained cotyledons, we have shown that epidermal cells of Arabidopsis cotyledons have more LDs as compared to parenchyma cells. As in Arabidopsis MM1 culture, presence of highly autofluorescent chloroplasts of green tissues did not pose any signal crosstalk issues while detecting LDs using Ac chemicals.

Taken together, these new fluorochromes and their possible future derivatives represent an attractive option for researchers to better understand in vivo behavior and biology of plant LDs.

Acknowledgments: We would like to thank Zsuzsanna Kószó and Katalin Török for technical assistance during experiments.

Conflict of interest: The authors declare that there are no conflicts of interest.

Author Contribution Statement: S.K. performed the experimental work. L.G.P. synthesized the Ac chemicals. F.A. designed and coordinated the project.

\section{References:}

Baud, S., \& Lepiniec, L. (2010). Physiological and developmental regulation of seed oil production. Progress in Lipid Research. doi:10.1016/j.plipres.2010.01.001

Chapman, K. D., Dyer, J. M., \& Mullen, R. T. (2012). Biogenesis and functions of lipid droplets in plants: Thematic Review Series: Lipid Droplet Synthesis and Metabolism: from Yeast to Man. Journal of Lipid Research, 53(2), 215-26. doi:10.1194/jlr.R021436

Chapman, K. D., \& Ohlrogge, J. B. (2012). Compartmentation of triacylglycerol accumulation in plants. Journal of Biological Chemistry. doi:10.1074/jbc.R111.290072

Cheng, J., Fujita, A., Ohsaki, Y., Suzuki, M., Shinohara, Y., \& Fujimoto, T. (2009). Quantitative electron microscopy shows uniform incorporation of triglycerides into existing lipid droplets. Histochemistry and Cell Biology, 132, 281-291. doi:10.1007/s00418-009-0615-z

Chudakov, D. M., Lukyanov, S., \& Lukyanov, K. A. (2007). Tracking intracellular protein movements using photoswitchable fluorescent proteins PS-CFP2 and Dendra2. Nature Protocols, 2, 2024-2032. doi:10.1038/nprot.2007.291

Coca, M., \& San Segundo, B. (2010). AtCPK1 calcium-dependent protein kinase mediates pathogen resistance in Arabidopsis. The Plant Journal $\square$ : For Cell and Molecular Biology, 63(3), 526-540. doi:10.1111/j.1365-313X.2010.04255.x

Cooper, M. S., Hardin, W. R., Petersen, T. W., \& Cattolico, R. A. (2010). Visualizing "green oil" in live algal cells. Journal of Bioscience and Bioengineering, 109(2), 198-201. doi:10.1016/j.jbiosc.2009.08.004 
De Kroon, A. I. P. M. (2007). Metabolism of phosphatidylcholine and its implications for lipid acyl chain composition in Saccharomyces cerevisiae. Biochimica et Biophysica Acta - Molecular and Cell Biology of Lipids. doi:10.1016/j.bbalip.2006.07.010

Digel, M., Ehehalt, R., \& Füllekrug, J. (2010). Lipid droplets lighting up: Insights from live microscopy. FEBS Letters. doi:10.1016/j.febslet.2010.03.035

Fasano, J. M., Swanson, S. J., Blancaflor, E. B., Dowd, P. E., Kao, T. H., \& Gilroy, S. (2001). Changes in root cap $\mathrm{pH}$ are required for the gravity response of the Arabidopsis root. The Plant Cell, 13, $907-$ 921. doi:10.1105/tpc.13.4.907

Fujimoto, T., Ohsaki, Y., Cheng, J., Suzuki, M., \& Shinohara, Y. (2008). Lipid droplets: a classic organelle with new outfits. Histochemistry and Cell Biology, 130(2), 263-79. doi:10.1007/s00418008-0449-0

Gáspár, I., \& Szabad, J. (2009). In vivo analysis of MT-based vesicle transport by confocal reflection microscopy. Cell Motility and the Cytoskeleton, 66, 68-79. doi:10.1002/cm.20334

Gidda, S. K., Shockey, J. M., Falcone, M., Kim, P. K., Rothstein, S. J., Andrews, D. W., ... Mullen, R. T. (2011). Hydrophobic-Domain-Dependent Protein-Protein Interactions Mediate the Localization of GPAT Enzymes to ER Subdomains. Traffic, 12, 452-472. doi:10.1111/j.1600-0854.2011.01160.x

Giepmans, B. N. G., Adams, S. R., Ellisman, M. H., \& Tsien, R. Y. (2006). The fluorescent toolbox for assessing protein location and function. Science (New York, N.Y.), 312, 217-224. doi:10.1126/science. 1124618

Greenspan, P., Mayer, E. P., \& Fowler, S. D. (1985). Nile red: A selective fluorescent stain for intracellular lipid droplets. Journal of Cell Biology, 100, 965-973. doi:10.1083/jcb.100.3.965

Grefen, C., Städele, K., Růžička, K., Obrdlik, P., Harter, K., \& Horák, J. (2008). Subcellular localization and in vivo interactions of the Arabidopsis thaliana ethylene receptor family members. Molecular Plant, 1, 308-320. doi:10.1093/mp/ssm015

Haraguchi, T., Shimi, T., Koujin, T., Hashiguchi, N., \& Hiraoka, Y. (2002). Spectral imaging fluorescence microscopy. Genes to Cells $\square$ : Devoted to Molecular \& Cellular Mechanisms, 7, 881887.

He, Y. Q., \& Wu, Y. (2009). Oil body biogenesis during Brassica napus embryogenesis. Journal of Integrative Plant Biology, 51, 792-799. doi:10.1111/j.1744-7909.2009.00851.x

Herman, E. M. (2008). Endoplasmic reticulum bodies: solving the insoluble. Current Opinion in Plant Biology. doi:10.1016/j.pbi.2008.08.004

Hsieh, K., \& Huang, A. H. C. (2004). Endoplasmic reticulum, oleosins, and oils in seeds and tapetum cells. Plant Physiology, 136, 3427-3434. doi:10.1104/pp.104.051060

Hsieh, K., \& Huang, A. H. C. (2007). Tapetosomes in Brassica tapetum accumulate endoplasmic reticulum-derived flavonoids and alkanes for delivery to the pollen surface. The Plant Cell, 19, 582596. doi:10.1105/tpc. 106.049049 
Kotogány, E., Dudits, D., Horváth, G. V, \& Ayaydin, F. (2010). A rapid and robust assay for detection of S-phase cell cycle progression in plant cells and tissues by using ethynyl deoxyuridine. Plant Methods, 6(1), 5. doi:10.1186/1746-4811-6-5

Lersten, N. R., Czlapinski, A. R., Curtis, J. D., Freckmann, R., \& Horner, H. T. (2006). Oil bodies in leaf mesophyll cells of angiosperms: Overview and a selected survey. American Journal of Botany, 93, 1731-1739. doi:10.3732/ajb.93.12.1731

Melo, R. C. N., D’Avila, H., Wan, H.-C., Bozza, P. T., Dvorak, A. M., \& Weller, P. F. (2011). Lipid bodies in inflammatory cells: structure, function, and current imaging techniques. The Journal of Histochemistry and Cytochemistry $\square$ : Official Journal of the Histochemistry Society, 59, 540-556. doi: $10.1369 / 0022155411404073$

Miquel, M., Trigui, G., d'Andréa, S., Kelemen, Z., Baud, S., Berger, A., ... Dubreucq, B. (2014). Specialization of oleosins in oil body dynamics during seed development in Arabidopsis seeds. Plant Physiology, 164, 1866-78. doi:10.1104/pp.113.233262

Murphy, D. J. (2001). The biogenesis and functions of lipid bodies in animals, plants and microorganisms. Progress in Lipid Research. doi:10.1016/S0163-7827(01)00013-3

Nan, X., Cheng, J.-X., \& Xie, X. S. (2003). Vibrational imaging of lipid droplets in live fibroblast cells with coherent anti-Stokes Raman scattering microscopy. Journal of Lipid Research, 44, 2202-2208. doi:10.1194/jlr.D300022-JLR200

Ohsaki, Y., Shinohara, Y., Suzuki, M., \& Fujimoto, T. (2010). A pitfall in using BODIPY dyes to label lipid droplets for fluorescence microscopy. Histochemistry and Cell Biology, 133(4), 477-80. doi:10.1007/s00418-010-0678-x

Purkrtova, Z., Jolivet, P., Miquel, M., \& Chardot, T. (2008). Structure and function of seed lipid bodyassociated proteins. Comptes Rendus - Biologies. doi:10.1016/j.crvi.2008.07.016

Puskás, L. G., Fehér, L. Z., Vizler, C., Ayaydin, F., Rásó, E., Molnár, E., ... Kitajka, K. (2010). Polyunsaturated fatty acids synergize with lipid droplet binding thalidomide analogs to induce oxidative stress in cancer cells. Lipids in Health and Disease, 9, 56. doi:10.1186/1476-511X-9-56

Rinne, P. L. H., Kaikuranta, P. M., \& Van Schoot, C. Der. (2001). The shoot apical meristem restores its symplasmic organization during chilling-induced release from dormancy. Plant Journal, 26, 249264. doi:10.1046/j.1365-313X.2001.01022.x

Slocombe, S. P., Cornah, J., Pinfield-Wells, H., Soady, K., Zhang, Q., Gilday, A., ... Graham, I. A. (2009). Oil accumulation in leaves directed by modification of fatty acid breakdown and lipid synthesis pathways. Plant Biotechnology Journal, 7, 694-703. doi:10.1111/j.14677652.2009.00435.x

Somwar, R., Roberts, C. T., \& Varlamov, O. (2011). Live-cell imaging demonstrates rapid cargo exchange between lipid droplets in adipocytes. FEBS Letters, 585, 1946-1950. doi:10.1016/j.febslet.2011.05.016 
Terskikh, A., Fradkov, A., Ermakova, G., Zaraisky, A., Tan, P., Kajava, A. V, ... Siebert, P. (2000). "Fluorescent timer": protein that changes color with time. Science (New York, N.Y.), 290, 15851588. doi: $10.1126 /$ science. 290.5496 .1585

Truernit, E., \& Haseloff, J. (2008). A simple way to identify non-viable cells within living plant tissue using confocal microscopy. Plant Methods, 4, 15. doi:10.1186/1746-4811-4-15

Tsien, R. Y. (2009). Constructing and exploiting the fluorescent protein paintbox (Nobel Lecture). Angewandte Chemie (International Ed. in English), 48, 5612-5626. doi:10.1002/anie.200901916

Van der Schoot, C., \& Rinne, P. L. H. (2011). Dormancy cycling at the shoot apical meristem: Transitioning between self-organization and self-arrest. Plant Science, 180, 120-131. doi:10.1016/j.plantsci.2010.08.009

Watanabe, T., Thayil, A., Jesacher, A., Grieve, K., Debarre, D., Wilson, T., ... Srinivas, S. (2010). Characterisation of the dynamic behaviour of lipid droplets in the early mouse embryo using adaptive harmonic generation microscopy. BMC Cell Biology, 11, 38. doi:10.1186/1471-2121-11-38

\section{Figure Legends}

Fig. 1: Lipid droplet labeling efficiency and analysis of cell viability using various treatment durations and doses of Ac chemicals on rice culture. Short $(15 \mathrm{~min})$ and long $(24 \mathrm{~h})$ treatment durations using $1,10,50 \mu \mathrm{M}$ of Ac chemicals (a-r) and DMSO (s-v) are shown. Propidium iodide (PI) labeling (in red) indicates dead cells. PI labeled untreated $2 \mathrm{~d}$ old and $9 \mathrm{~d}$ old control cultures are shown in w, x. Single plane confocal fluorescence images are shown. Cyan (Ac chemical) and red (PI) fluorescence emissions are merged with transmission images. Identical excitation energy and fluorescence emission sensitivity settings were used for all images. Scale bar $20 \mu \mathrm{m}$

Fig. 2: (a-c) Ac chemicals and LD dye Nile Red colocalize completely in cultured rice cells in vivo. Ac chemicals (cyan) and Nile Red (red) single optical section images are overlaid onto bright field images on the last column. Insets show enlarged images of rectangular selection areas. Due to differences in emission intensities of Ac chemicals, varying detector sensitivities (Ac-202<Ac-201<Ac-1041=DMSO) were used for capturing each image. To assess the degree of crosstalk between blue and red channels, single dye labeled control cells were imaged in both channels and displayed as composite images (d). DMSO (DM) is used as solvent control. Scale bar is $20 \mu \mathrm{m}$

Fig. 3: Comparison of spectral emission characteristics of Ac chemicals and Nile Red using spectral imaging and live cell microscopy on rice cell cultures. Average fluorescence emission intensities of dye labeled LDs are plotted following spectral imaging with a confocal microscope. Emission ranges (above 20\% intensity) of EYFP (Enhanced yellow fluorescent protein), dsRed, mCherry and chlorophyll $\mathrm{b}$ are indicated as colored rectangles to show the degree of spectral overlap with Nile Red emission. Representative spectral image series of LDs stained with Ac dyes (detection: 410-510 nm) and Nile Red (detection: 500-730 nm) are shown at the bottom aligned with the abscissa of the chart (wavelength in $\mathrm{nm}$ ). LDs of three different cells were used for calculating averages and standard deviations. Each image frame below the chart is $4.4 \mu \mathrm{m}$ wide

Fig. 4: Lipid droplet detection on various plant cultures using Ac chemicals. LD labeling with Ac-201, Ac-202 and Ac-1041 chemicals (10 $\mu \mathrm{M}, 15 \mathrm{~min})$ were tested on (a-c) alfalfa (M. sativa ssp. varia A2), (d-f) maize (Z. mays, cv. H1233), (g-i) tobacco (N. tabacum cv. Petit Havana SR1) and (j-l) chlorophyll containing Arabidopsis $(A$. thaliana ecotype Landsberg erecta, MM1) cell cultures. Merged images of chemical fluorescence (cyan), chlorophyll (red) and bright field (grayscale) are shown. Insets show $3 x$ magnified close-up images of representative 
selected regions (white rectangles in $\mathbf{j}-\mathbf{l}$ ). Note complete color separation between cyan and red channels in insets. Scale bar $20 \mu \mathrm{m}$

Fig. 5: Lipid droplet labeling on seedlings using Ac chemicals. Arabidopsis (ecotype Columbia-0) seedlings were incubated $1 \mathrm{~h}(10 \mu \mathrm{M})$ with Ac-201 (a-d) and Ac-202 (e-f). In vivo LD detection on various plant parts are shown in a-d. Ac-202 treated seedling's cotyledon was optically sectioned using laser scanning confocal microscope (e-g). Zaxis projection (image merging) of 396 optical sections of cotyledon epidermis/upper parenchyma region is shown in e. Three dimensional reconstruction of merged $z$-axis images in $\mathbf{e}$ is shown in $\mathbf{f}$. For better visualization of 3D morphology, image in $\mathbf{f}$ is contrast enhanced to amplify faint cell wall fluorescence of epidermal cells. $\mathrm{Y}$-axis projection (side view) of additional 396 scans shown in (g) was obtained by fast scanning of a smaller area (yellow rectangular area in $\mathbf{e})$. White and yellow arrows (e-g) mark two stomata which also indicate orientation of 3D image. Scalebars in a-d are $20 \mu \mathrm{m}$ and interstomatal distance (center to center) is $74 \mu \mathrm{m}$ in $\mathbf{e}-\mathbf{g}$

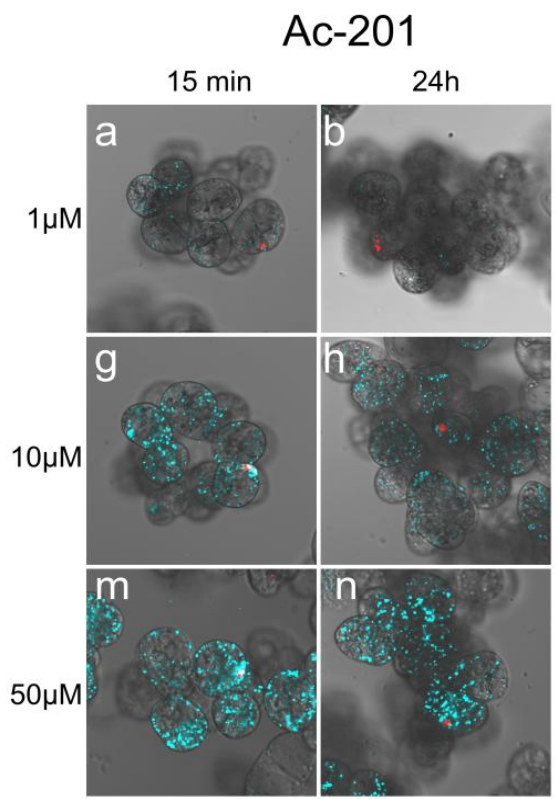

DMSO $1 \%$

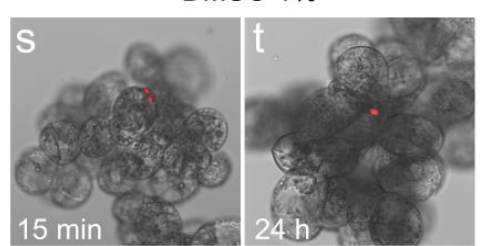

Ac-202

$15 \min$
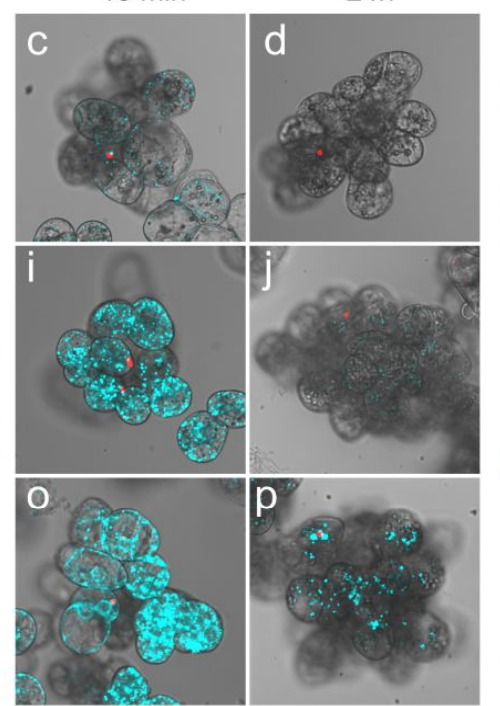

DMSO $10 \%$

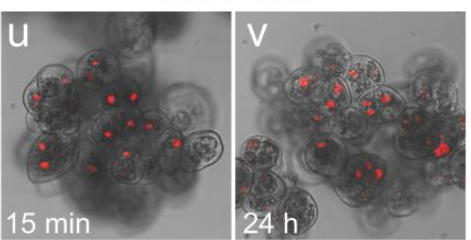

Fig. 1

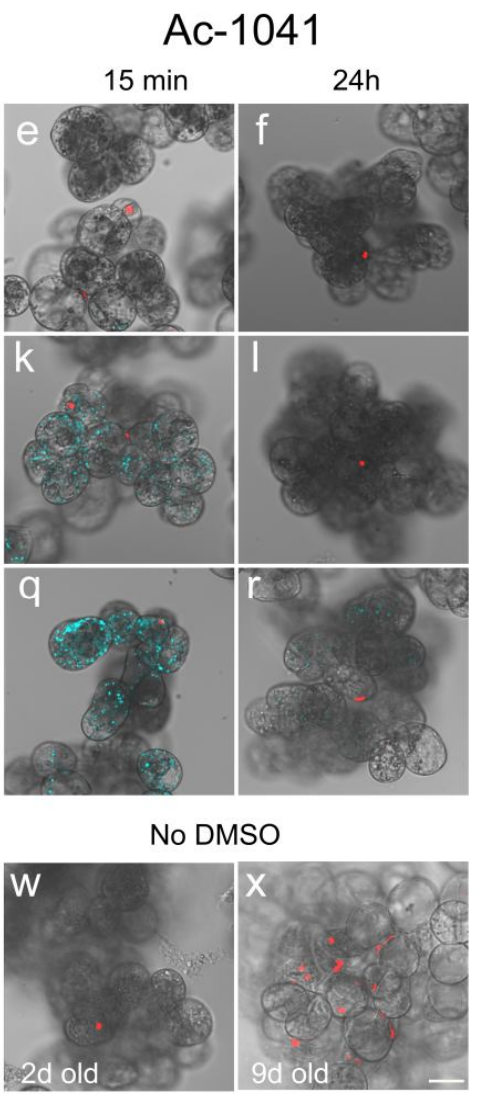




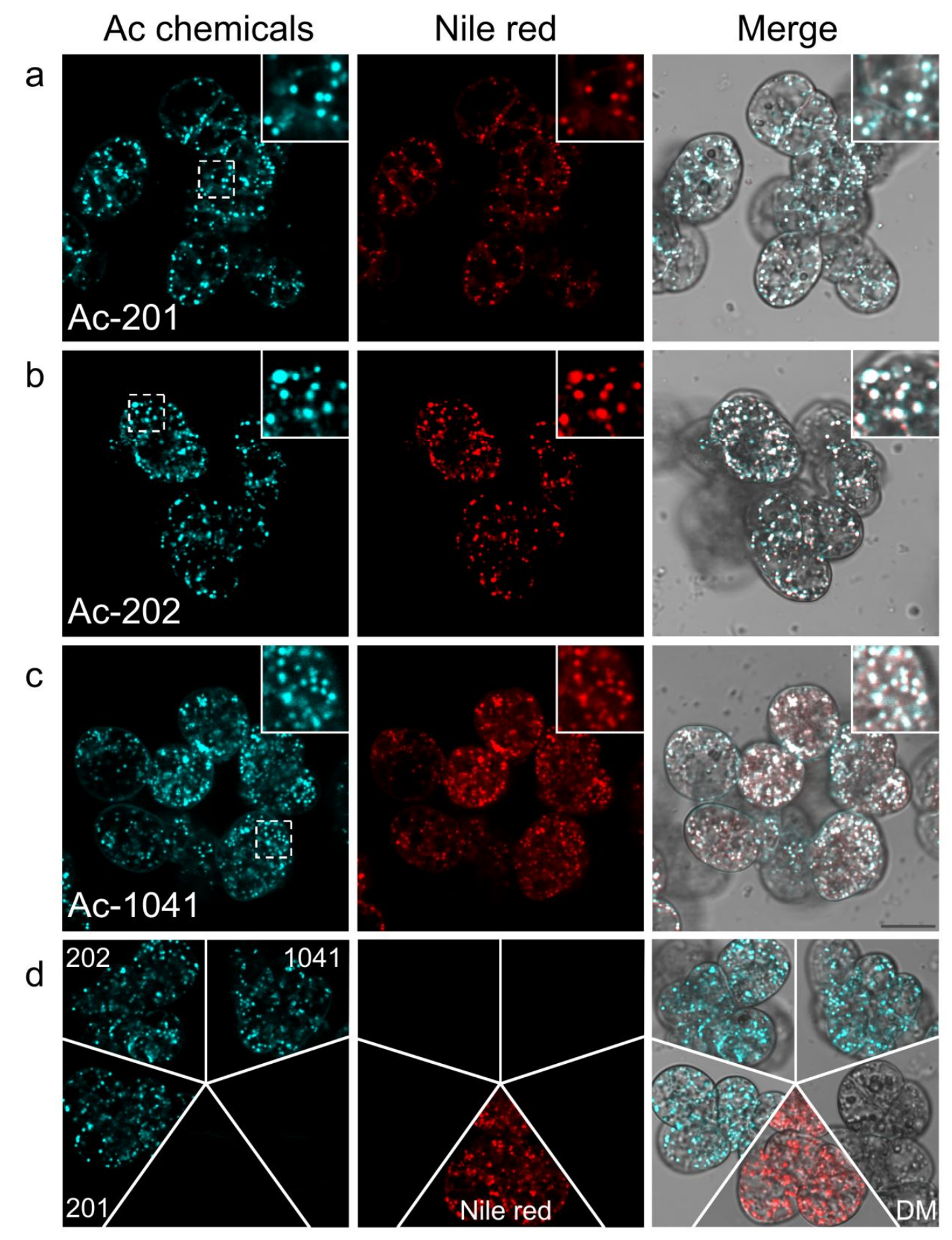

Fig. 2 


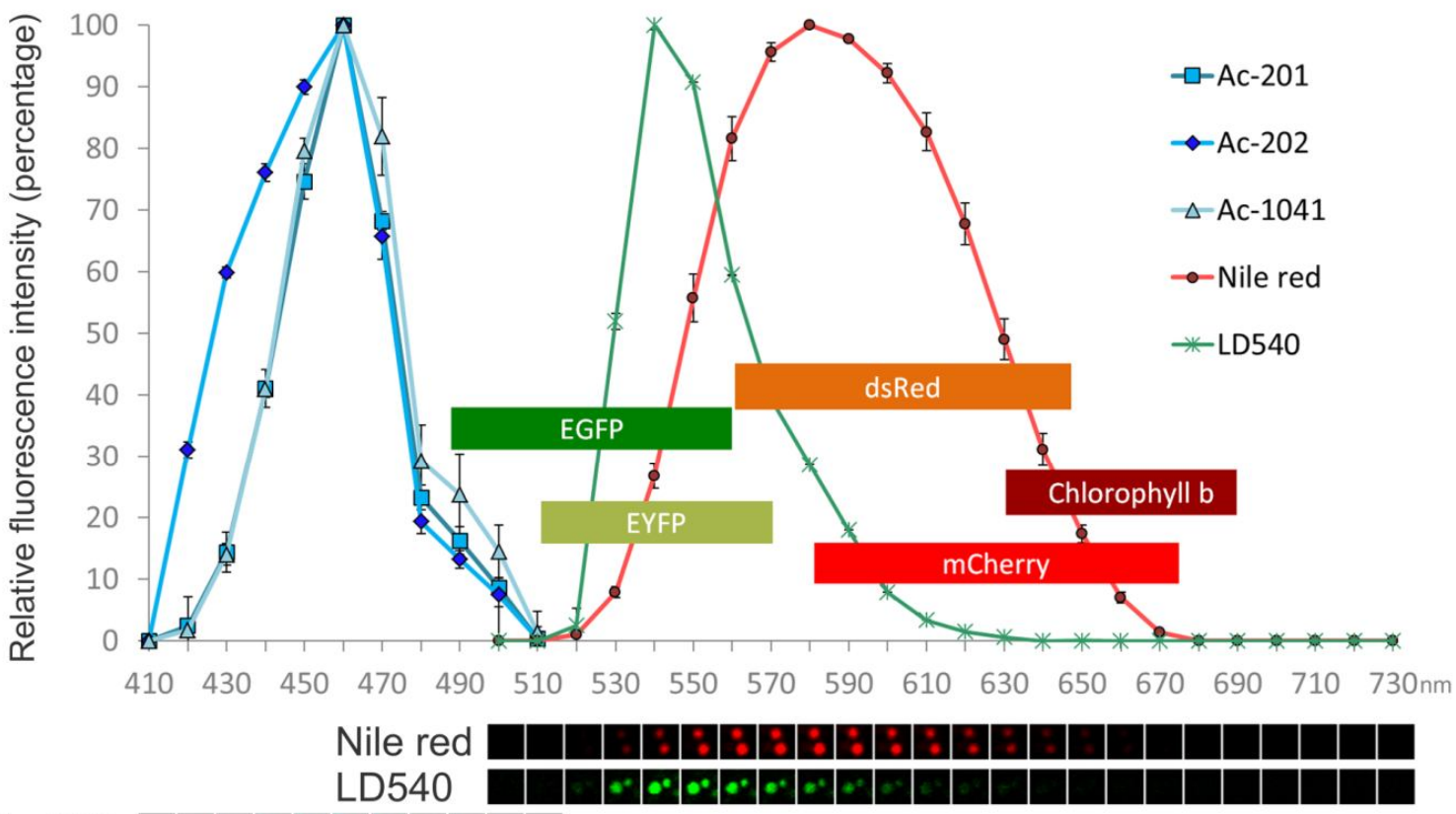

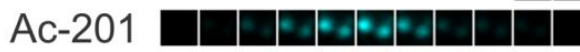

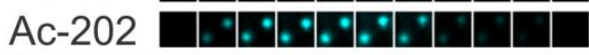

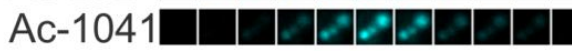

Fig. 3 

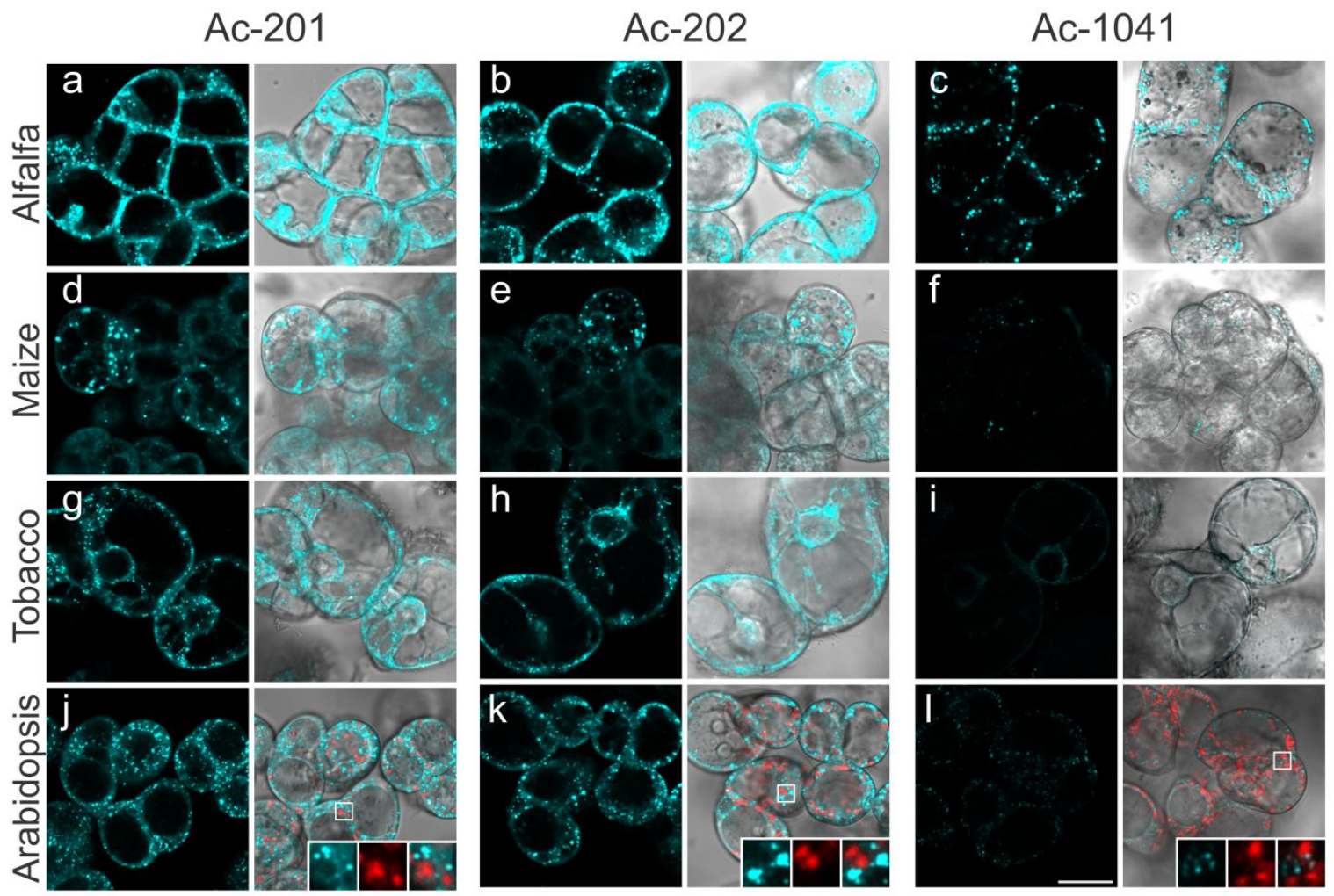

Fig. 4 


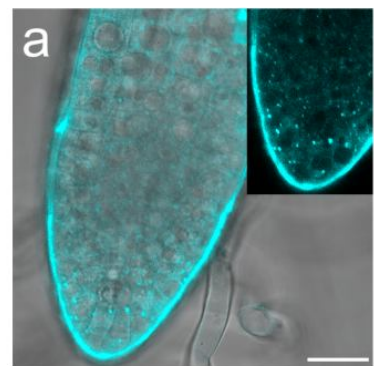

Root tip

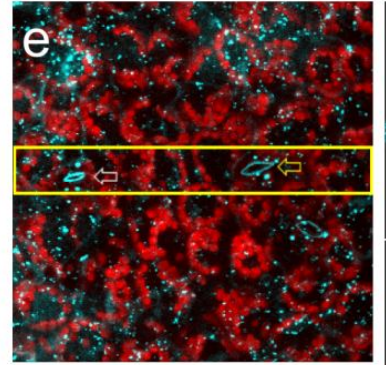

Z-axis projection

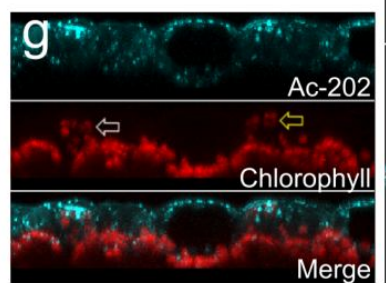

Y-axis projection

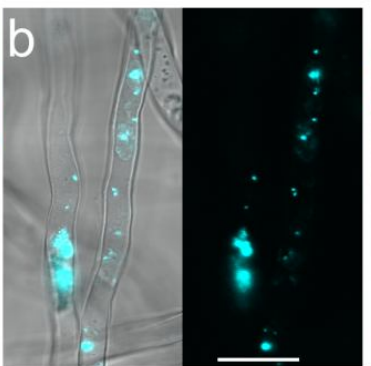

Root hairs

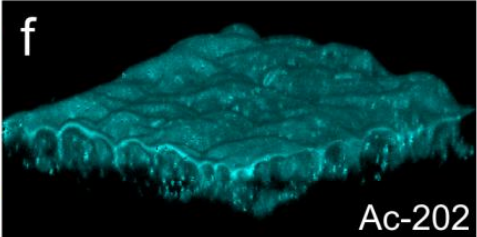

Ac-202

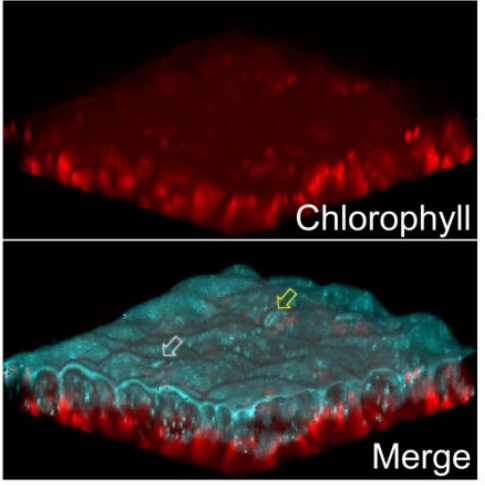

$3 \mathrm{D}$ reconstruction

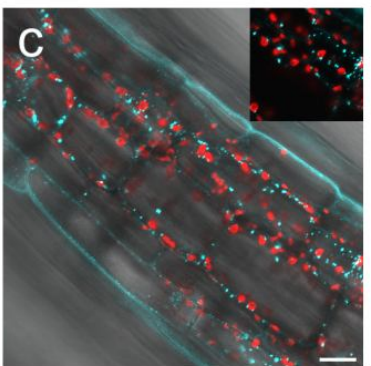

Hypocotyl

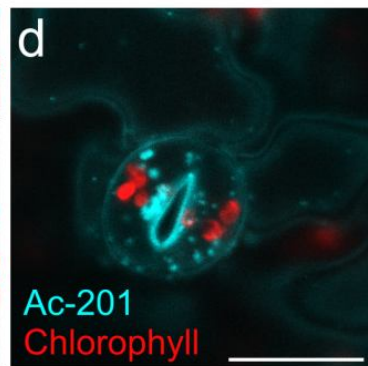

Stoma/Epidermis

\section{Fig. 5}

\section{Supplementary Figure Legends}

\section{Supplementary Figure 1}

Ac-201

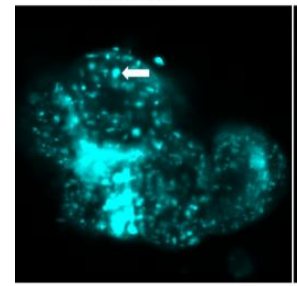

EGFP

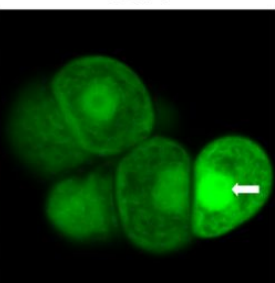

Propidium lodide

Merge1

Merge2

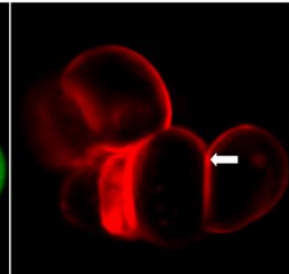

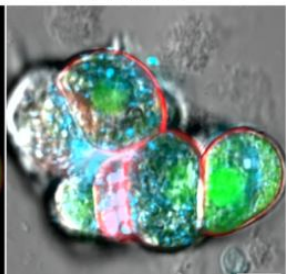

Detection of oil bodies (Ac-201), enhanced green fluorescent protein (EGFP) and cell borders/cell walls (propidium iodide) on live rice cell cultures (O. sativa L. ssp. japonica cv.'Nipponbare') stably expressing untagged EGFP protein. Arrows mark representative high signal intensity regions in cyan, green and red 
channels. Note the absence of channel crosstalk in these regions. Three-color merged image (Merge 1) was overlaid onto bright field image at the last panel (Merge 2). Scale bar is $10 \mu \mathrm{m}$.

\section{Supplementary Figure 2}

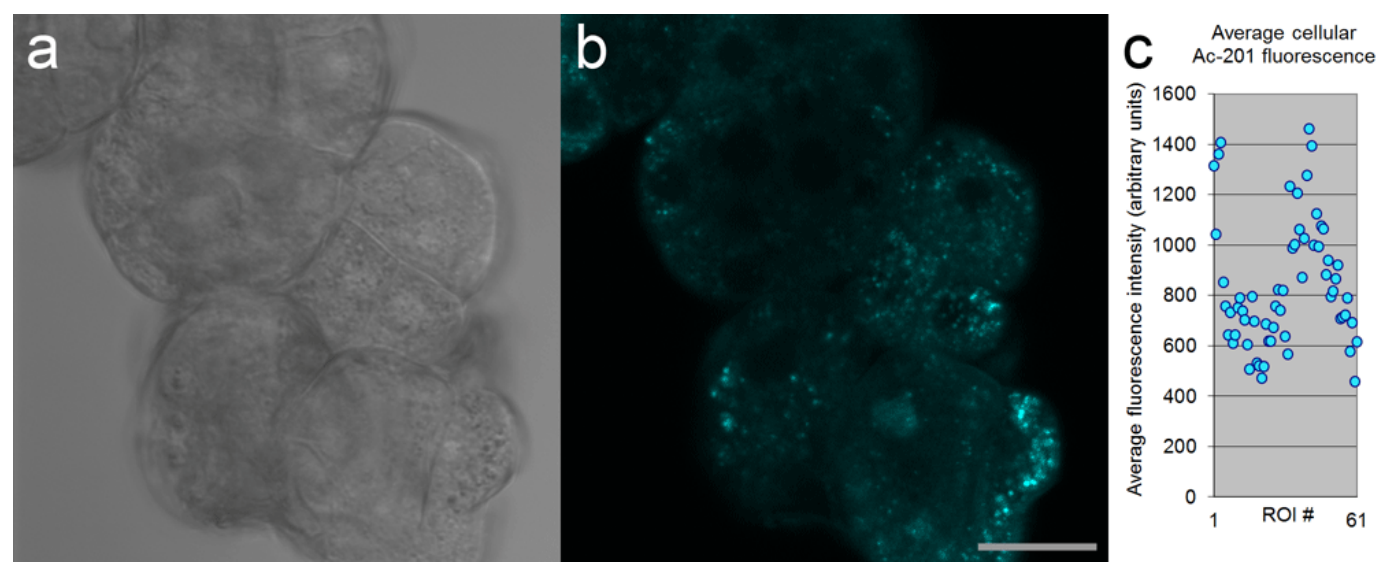

Animated (gif) z-stack image series of bright field (a) and Ac-201 stained fluorescence (b) images of maize culture (Z. mays, cv. H1233) showing lipid droplet heterogeneity within a cell cluster. Scale bar $20 \mu \mathrm{m}$. Chart in (c) shows average cellular fluorescence intensity distribution of 61 cells from 4 different cell clusters. Intensity measurements were performed on merged $\mathrm{z}$ sections using maximum intensity projection of individual optical sections.

\section{Supplementary Figure 3}
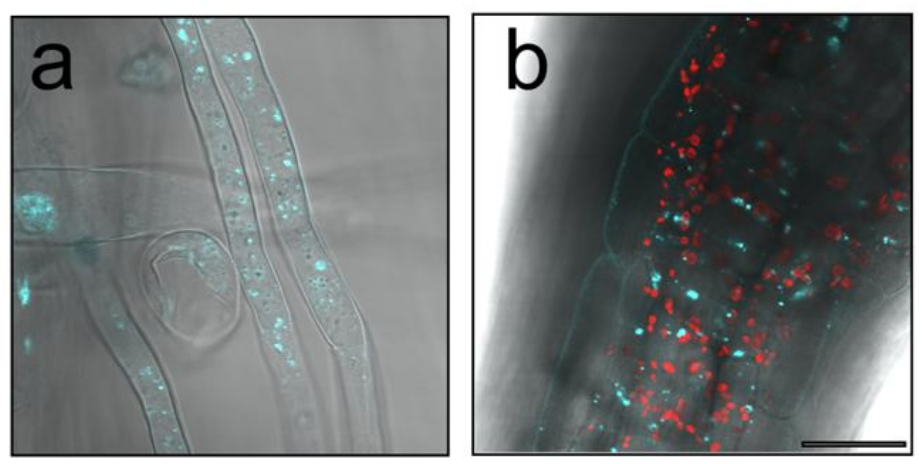

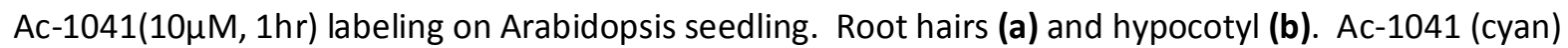
and chlorophyll (red) images are overlaid onto bright field images in (b). Scale bar $20 \mu \mathrm{m}$

\section{Supplementary Figure 4.}




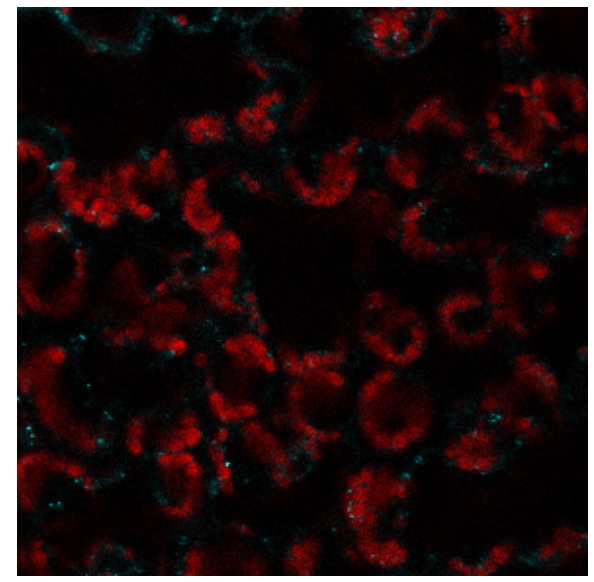

Animated (gif) z-stack image series of Ac-201 stained (cyan) Arabidopsis cotyledon showing dye penetration to parenchyma cells containing chloroplasts (red). Image dimensions in $\mathrm{x}, \mathrm{y}$ and $\mathrm{z}$ axes are $147 \mu \mathrm{m}, 147 \mu \mathrm{m}$ and $30 \mu \mathrm{m}$, respectively.

\section{Supplementary Figure 5.}

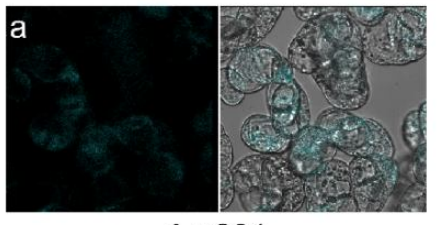

Ac-201

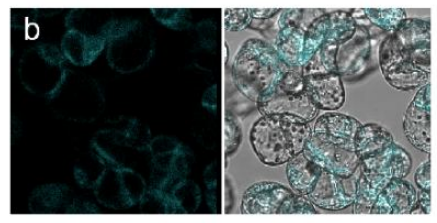

Ac-202

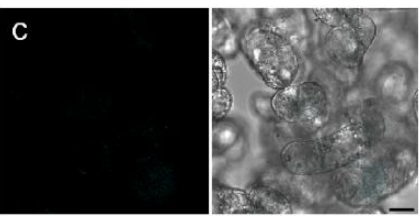

Ac-1041

Application of low concentration ( $1 \mu \mathrm{M}, 15$ mins) of Ac chemicals on alfalfa cultures. (a) Ac-201, (b) Ac202, (c) Ac-1041. Scale bar is $20 \mu \mathrm{m}$. 